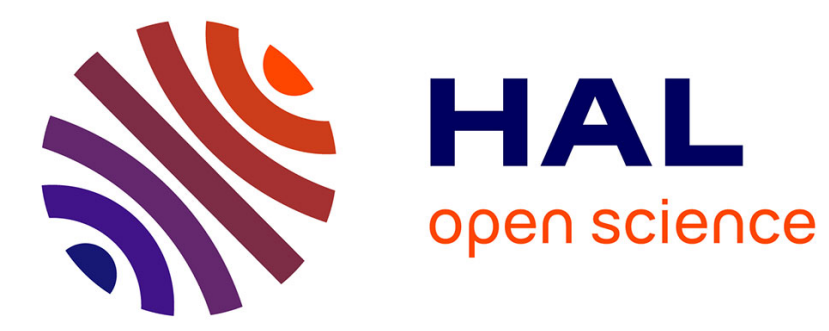

\title{
Abstract Concept Lattices
}

\author{
Henry Soldano, Veronique Ventos
}

\section{To cite this version:}

Henry Soldano, Veronique Ventos. Abstract Concept Lattices. International Conference on Formal Concept Analysis, May 2011, Nicosia, Cyprus. pp.235-250, 10.1007/978-3-642-20514-9_18 . hal00642105

\section{HAL Id: hal-00642105 \\ https://hal.inria.fr/hal-00642105}

Submitted on 17 Nov 2011

HAL is a multi-disciplinary open access archive for the deposit and dissemination of scientific research documents, whether they are published or not. The documents may come from teaching and research institutions in France or abroad, or from public or private research centers.
L'archive ouverte pluridisciplinaire HAL, est destinée au dépôt et à la diffusion de documents scientifiques de niveau recherche, publiés ou non, émanant des établissements d'enseignement et de recherche français ou étrangers, des laboratoires publics ou privés. 


\title{
Abstract Concept Lattices
}

\author{
Henry Soldano ${ }^{1}$, Véronique Ventos ${ }^{2}$ \\ 1 L.I.P.N, UMR-CNRS 7030, Université Paris-Nord, \\ 93430 Villetaneuse, France \\ 2 LRI, UMR-CNRS 8623, Université Paris-Sud, \\ 91405 Orsay, France \\ henry.soldano@ipn.univ-paris13.fr, ventos@lri.fr
}

\begin{abstract}
We present a view of abstraction based on a structure preserving reduction of the Galois connection between a language $\mathcal{L}$ of terms and the powerset of a set of instances $O$. Such a relation is materialized as an extension-intension lattice, namely a concept lattice when $L$ is the powerset of a set $P$ of attributes. We define and characterize an abstraction $A$ as some part of either the language or the powerset of $O$, defined in such a way that the extension-intension latticial structure is preserved. Such a structure is denoted for short as an abstract lattice. We discuss the extensional abstract lattices obtained by so reducing the powerset of $O$, together together with the corresponding abstract implications, and discuss alpha lattices as particular abstract lattices. Finally we give formal framework allowing to define a generalized abstract lattice whose language is made of terms mixing abstract and non abstract conjunctions of properties.
\end{abstract}

\section{Introduction}

There were in machine learning various attempts to formalize abstraction and characterize its desirable properties with respect to induction. An important statement was that abstraction should be order-preserving with respect to the partially ordered language in which hypotheses are searched for [21]. However, the question of what classes of abstractions are to be investigated for learning and reasoning is far from being exhausted. We present a view of abstraction based on a structure preserving reduction of the relation between a term $t$ of a language $\mathcal{L}$, partially ordered following a general-to-specific partial order, and the extension of $t$ on a set of objects (or instances) $O$, representing the subset of $O$ whose elements satisfy the term.

In Formal Concept Analysis [15] and Galois lattice theory [4] $\mathcal{L}$ is a lattice, and the relation between $\mathcal{L}$ and the powerset $\mathcal{P}(O)$ is materialized as an extension-intension lattice. This lattice is the structure of the definable elements of $\mathcal{P}(O)$ [1], i.e. the subsets of $O$ that are each the extension of some term of $\mathcal{L}$. In such a lattice, a definable set $e$ represents the equivalence class of all the terms whose extension is $e$, and a node, also called a concept, is a pair $(e, t)$ where $t$ is the most specific term of the class, denoted as the intension of the concept. Formal Concept Analysis is primarily concerned with the relation between the powerset of a set of properties as a language and $\mathcal{P}(O)$. The extension-intension lattice is then denoted as a concept lattice. Various extensions have been recently proposed to ease the representation in more sophisticated languages [6, 
18]. In particular pattern structures [16] have been recently introduced to represent complex data, associating such a pattern structure to each object. Logical Concept Analysis (LCA) [13] has been recently introduced as a general formalization in which $\mathcal{L}$ is a logical language and uses object descriptions in $\mathcal{L}$. Though we do not use here the LCA formulation and notations, for technical reasons, our construction of a Galois connection on a modal language is very similar to the construction presented in [12]. In a recent paper [20] particular mappings, denoted as projections, are used to reduce $\mathcal{L}$ or $\mathcal{P}(O)$ in such a way that the relation between the language and the extensional space is still materialized as a lattice. In other words, projections ensure that we have a coarser, yet structure preserving, view of concepts representatives of the universe we deal with. Independently [16] also uses projections on pattern structures.

In this paper we show that projections of a lattice are in a one to one correspondence with abstractions defined as parts of the lattice that are closed under least upper bound. We call the corresponding structures abstract extension-intension lattices and abstract concept lattice, and for short abstract lattices. We first briefly discuss intensional abstractions and investigate then more specifically extensional abstractions, i.e. parts of $\mathcal{P}(O)$ that are closed under set theoretic union.

More precisely, applying an extensional abstraction means that we will no longer consider instances of $O$ as elements of the extensional space, but rather consider subsets of $O$ given a priori. As an example consider $O=\left\{o_{1}, o_{2}, o_{3}, o_{4}\right\}$, and $A$ be obtained by closing under union the part $\left\{\left\{o_{1}, o_{2}\right\},\left\{o_{1}, o_{3}\right\}\right\}$. As a result, $\left\{o_{1}, o_{2}, o_{3}\right\}$ belongs to $A$ but $\left\{o_{2}, o_{3}\right\}$ does not. Now, consider the smallest elements of $A$ that contain a given instance $o$. We call these elements the minimal abstractions of $o$ and consider them as abstract instances. In this example, $\left\{o_{1}, o_{2}\right\}$ is the unique minimal abstraction of $o_{2},\left\{o_{1}, o_{3}\right\}$ is the unique minimal abstraction of $o_{3},\left\{o_{1}, o_{2}\right\}$ and $\left\{o_{1}, o_{3}\right\}$ are the two minimal abstractions of $o_{1}$, and $o_{4}$ has no abstraction in $A$.

We relate then any term $t$ to an abstract extension $\operatorname{ext}_{A}(t)$ that turns out to be the union of all the abstract instances included in $\operatorname{ext}(t)$. Going back to our example, suppose that $\operatorname{ext}(t)=\left\{o_{1}, o_{2}, o_{3}, o_{4}\right\}$, then the abstract instances included in $\operatorname{ext}(t)$ are $\left\{o_{1}, o_{2}\right\}$ and $\left\{o_{1}, o_{3}\right\}$ and therefore $\operatorname{ext}_{A}(t)=\left\{o_{1}, o_{2}, o_{3}\right\}$. Clearly the abstract extension of a given term is always included in its original extension. In other words we reduce the extension of the term by excluding any instance that has no minimal abstraction included in the original extension. In the current example, $o_{4}$ is such an instance. The intuition here is a change in granularity: the new objects we deal with are the minimal abstractions of the original instances. Furthermore, as an extension-intension lattice represents a set of valid implications (see for instance [4]), our abstract extension-intension lattice also represents a set of valid abstract implications. Going back to our example, suppose $\operatorname{ext}(p)=\left\{o_{1}, o_{2}, o_{4}\right\}$ and $\operatorname{ext}(q)=\left\{o_{1}, o_{2}, o_{3}\right\}$, then the implication $p \rightarrow q$ is not valid on $O$. However, as we have $\operatorname{ext}_{A}(p)=\left\{o_{1}, o_{2}\right\}$ and $\operatorname{ext}_{A}(q)=\left\{o_{1}, o_{2}, o_{3}\right\}$, the abstract implication $p \rightarrow_{A} q$ is valid. As a matter of fact a general property of extensional abstractions is that they preserve validity of implications. Algorithms that extract valid implications $[14,17]$ from a set of instances $O$, can be extended to extract valid abstract implications. As an example, we can interpret alpha lattices [28] as particular abstract lattices, and alpha implications are straightforwardly extracted by extending the method of N. Pasquier and collaborators [19] to alpha lattices. 
Regarding extensional abstractions, the formal work closest to ours regards rough sets logics. Rough sets originally relies on an indiscernibility relation (see for instance [29]). More recently, generalization of rough sets using coverings, corresponding to our extensional abstractions, has been investigated from an algebraic perspective [5, 8]. However these works do not investigate the extension-intension relationship.

To summarize, in this first part of the paper we characterize and discuss the properties of abstractions, and particularly of extensional abstractions, as a structure and order preserving reduction of extension-intension lattices.

We then remark that abstract implications are in fact implications between abstract terms, i.e. $t_{1} \rightarrow t_{2}$ rewrites as $\square t_{1} \rightarrow \square t_{2}$, where $\square t$ means "Abstractly $t_{\text {" with respect }}$ to the abstraction $A$. Then, starting from the semantics, we discuss the corresponding abstract modal logics. This leads us to consider a language $\mathcal{L} \square$ a term of which is made of a non abstract part together with an abstract part and to define a new, more expressive, extension-intension lattice.

\section{Preliminaries}

\subsection{Galois Connection}

We recall here the definitions of a Galois connection and a Galois lattice.

Definition 1 Given two lattices $\left(E, \leq_{E}, \wedge, \vee\right)$ and $\left(F, \leq_{F}, \wedge, \vee\right)$, where $\leq_{E}$ and $\leq_{F}$ denote the order relations, and $\wedge, \vee$ the meet and the join operations, a Galois connection between $E$ and $F$ is a pair of mappings $(f: E \rightarrow F, g: F \rightarrow E)$ verifying the following properties:

- For any $x$ and $x^{\prime}$ in $E$, we have that $x \leq_{E} x^{\prime}$ implies $f(x) \geq_{F} f\left(x^{\prime}\right)$

- For any $y$ and $y^{\prime}$ in $F$, we have that $y \leq_{F} y^{\prime}$ implies $g(y) \geq_{E} g\left(y^{\prime}\right)$

- For any $x$ in $E$ and $y$ in $F$ we have that $g \circ f(x) \geq_{E} x$ and $f \circ g(y) \geq_{F} y$

The Galois lattice defined by the Galois connection $(f, g)$ is then the set $\{(x, y) \in$ $E \times F \mid y=f(x)$ and $x=g(y)\}$ ordered by $\leq_{E}$

\subsection{Extension-intension lattices}

FCA, in a broad sense, investigates the link between the terms of some language $\mathcal{L}$ and a universe $O$ of elements of the universe denoted as instances. We also call intensional representations the terms of $\mathcal{L}$ and extensional representations the elements of the powerset $\mathcal{P}(O)$. In this presentation we suppose that we know whenever an instance $o$ satisfies the term $t$, and we define accordingly its extension:

Definition $2 \operatorname{ext}_{O}(t)=\{o \in O \mid$ o satisfies $t\}$.

In what follows we consider $O$ as a fixed and finite set of instances and we simply write $\operatorname{ext}(t)$ the extension in $O$.

The language $\mathcal{L}$ is partially ordered by a general-to-specific relation. We write $t 1 \preceq$ $t 2$ whenever $t_{1}$ is less specific than $t_{2}$, or equivalently $t_{1}$ is more general than $t_{2}$. The following proposition relate $\mathcal{L}$ and $\mathcal{P}(O)$ by a Galois connection. 
Proposition 1 Let $\mathcal{L}$ be a finite language, $\preceq$ a partial order on $\mathcal{L}$ denoted as specificity, $O$ be a finite set of instances and ext $: \mathcal{L} \rightarrow \mathcal{P}(O)$ be a mapping such that $t_{1} \preceq t_{2} \Rightarrow$ $\operatorname{ext}\left(t_{1}\right) \supseteq \operatorname{ext}\left(t_{2}\right)$. We consider the following conditions:

- (condition 2.1) For any instance o, there is a unique most specific term, denoted as the object description $d(o)$, among all terms $t$ such that $o \in$ ext $(t)$

- (condition 2.2) $\mathcal{L}$ has a greatest element and is a lower semi lattice, i.e. each pair of terms $t_{1}, t_{2}$ of $\mathcal{L}$ has a unique greatest lower bound $t_{1} \wedge_{\mathcal{L}} t_{2}$ in $\mathcal{L}$, also called the least general generalisation (for short $\operatorname{lgg}$ ) of $t_{1}$ and $t_{2}$.

Whenever conditions 2.1 and 2.2 are satisfied, $(\mathcal{L}, \preceq)$ is a lattice, i.e. two terms $t_{1}$ and $t_{2}$ also have a least upper bound denoted $t_{1} \vee_{\mathcal{L}} t_{2}$, and the pair (int, ext) where

$$
\operatorname{int}(e)=\bigwedge_{o \in e_{\mathcal{L}}} d(o)
$$

is a Galois connection.

In Formal Concept Analysis [15] and Galois Analysis [4], $\mathcal{L}$ is the powerset of a set $P$ of attributes. Recent extensions to various languages have been performed [14, 13]. In particular in [16], $\mathcal{L}$ is defined as a set of pattern structures, i.e. terms generated by first considering the set of object descriptions, and then closing it under the least general generalization $\wedge_{\mathcal{L}}$. Independently, E. Diday and R. Emilion start from the same assumptions [9]. Proposition 1 directly follows from, for instance, theorem 2 in [9].

We call extension-intension lattice the Galois lattice $G$ coresponding to this Galois connection. $G$ is ordered using the extensional order and each element $G$ is a pair $(e, t)$ such that $t=\operatorname{int}(e)$ and $e=\operatorname{ext}(t)$, i.e. $t$ is the unique most specific term representing the equivalence class of terms whose extension is $e$, and is also denoted as the intension of $e$. Such most specific terms are also referred as closed terms or closed motifs in data mining [25]. In Machine Learning, the search space $\mathcal{L}$ may be in this way explored by minimally generalizing or specializing such closed terms [3].

Finally $G$ is considered as the structure of $O$ as perceived through $\mathcal{L}$ and can be also represented by the set $T_{O}$ of all the implications $p \rightarrow q$ which are valid on $O$, i.e. such that $\operatorname{ext}(p) \subseteq \operatorname{ext}(q)$ is true. The equivalence class whose intension is $t=\operatorname{int}(e)$, also contains various minimal (i.e. most general) terms $t^{m}$, also know as generators. The elements of $T_{O}$ can be generated from the set of all the $t^{m} \rightarrow t$ implications also called the min-max implications basis of $T_{O}$ [19].

\subsection{Projections and projected extension-intension lattices}

Now, consider the following problem; how to reduce $\mathcal{P}(O), \mathcal{L}$, or both, in such a way that $i$ ) conditions 2.1 and 2.2 are still satisfied, $i i)$ the resulting extension-intension lattice $G^{\prime}$ is isomorphic to part of $G$. In other words, how to reduce $G$ by reducing the language or the powerset of the universe of instances in such a way that the structure is preserved, and its size reduced ? An answer to this question is given in [20] through the use of intensional and extensional projections whose images are also lattices: 
Definition 3 (Projection) $p$ is a projection of a lattice $(M, \leq)$ iff for each pair $(x, y)$ of elements of $M$, we have :

If $x \leq y$ then $p(x) \leq p(y)$ (monotonicity)

$p(x) \leq x$ (minimality)

$p(x) \leq p(p(x))$ (semi-idempotence)

Let $M$ be a lattice and $p$ a projection of $M$, then $p(M)$ is also a lattice with join operator $\vee$ and meet operator $\wedge$ defined as, for any pair $m_{1}, m_{2} \in p(M), m_{1} \wedge m_{2}=$ $p\left(m_{1} \wedge_{M} m_{2}\right)$ and $m_{1} \vee m_{2}=m_{1} \vee_{M} m_{2}$.

When considering respectively $M=\mathcal{L}$ and $M=\mathcal{P}(O)$ we obtain respectively intensional and extensional projections and both lead to projected extension-intension lattices [20,28].

Proposition 2 Let (int, ext) be a Galois connection on $(\mathcal{P}(O), \preceq),(\mathcal{L}, \subseteq))$, G be the associated Galois lattice, and $(e, t)$ be a node of $G$.

- Let $p$ be a projection on $\mathcal{L}$, then $(p \circ$ int, ext) defines a Galois connection on $((p(\mathcal{L}), \preceq),(\mathcal{P}(O), \subseteq))$ and $(e, t)$ is projected in the corresponding Galois lattice $p(G)$ on the node $\left(e^{\prime}, t^{\prime}\right)$ such that $t^{\prime}=p(t)$ and $e^{\prime}=\operatorname{ext}\left(t^{\prime}\right)$.

- Let $p$ be a projection on $\mathcal{P}(O)$, then (int, $p \circ$ ext) defines a Galois connection on $((\mathcal{L}, \preceq),(p(\mathcal{P}(O)), \subseteq))$ and $(e, t)$ is projected in the corresponding Galois lattice $p(G)$ on the node $\left(e^{\prime}, t^{\prime}\right)$ such that $e^{\prime}=p(e)$ and $t^{\prime}=\operatorname{int}\left(e^{\prime}\right)$.

Note that in partial order theory, projections are known as kernel operators or interior operators. Their properties are well known [11] and are the basis of the next section.

\section{Abstractions}

\subsection{From projection to abstractions}

Reducing through projections $\mathcal{L}, \mathcal{P}(O)$ or both results in a reduced extension-intension representation whose latticial structure is preserved. However, while projections are technically useful, they do not always give a simple way to chose simplified representations. In what follows we present an equivalent view of projections.

Definition 4 An abstraction of a lattice $M$ is a subset ${ }^{3}$ of $M$, closed under $\vee_{M}$.

Building abstractions therefore simply means to chose any subset of $M$ and close it by $\vee_{M}$. We note hereunder that abstractions are in a one to one correspondence with projections and so abstract extension-intension lattices are defined as projected extensionintension lattices.

Proposition 3 Let $A$ be an abstraction of a lattice $M$, then $p_{A}$ defined as $p_{A}(x)=$ $\bigvee_{c \in A, c<x} c$, is a projection of $M$. Let $p$ be a projection then $A=p(M)$ is an abstraction of $\bar{M}$, and $p$ is the projection $p_{A}$ associated to $A$.

\footnotetext{
${ }^{3}$ Note that $\perp_{M}$, the smallest element of $M$, belongs to all abstractions.
} 
The equivalence between interior systems $p(M)$ and subsets $A$ of $M$ closed under union is a known property of interior operators [11]. As abstractions are closed under $\vee_{M}$, we have that $\vee_{M}=\vee_{A}$. From now on we simply write $\vee$ when no confusion is possible. An important point is that we only need the $\vee$-irreducible elements ${ }^{4}$ of $A$ :

Proposition 4 Let $A$ be an abstraction of $M$, and $A_{I}$ be the set of $\vee$-irreducible elements of $A$, the projection $p_{A}$ is obtained by only considering elements of $A_{I}$ :

$$
p_{A}(x)=\bigvee_{c \in A_{I}, c \leq x} c
$$

Intensional abstractions An intensional abstraction is then simply obtained by selecting part of the language $\mathcal{L}$ and closing it by the join operator $\vee_{\mathcal{L}}$. For instance, consider the lattice $\mathcal{L}$ whose elements are intervals $[a, b]$ such that $a, b \in\{1,2,3,4\}$. We have then $[1,3] \vee_{\mathcal{L}}[3,4]=[3]$. Consider first the abstraction $A$ whose elements are the intervals containing 3 . $A$ is closed by $\vee_{\mathcal{L}}$ as intersecting two intervals containing 3 results in an interval also containing 3.

Consider now $\mathcal{L}^{\prime}=\mathcal{L}-\{[1],[2],[3],[4]\} . \mathcal{L}^{\prime}$ is obtained by simply deleting the most specific terms and is clearly also a lattice. However $[1,3] \vee_{\mathcal{L}^{\prime}}[3,4]$ is now [] and so clearly $\mathcal{L}^{\prime}$ is not closed under $\vee_{\mathcal{L}}$, and therefore is not an abstraction. Note that there are in $\mathcal{L}^{\prime}$ two most specific elements satisfied by $o=3$, and as a consequence $d(o)$ is no longer defined.

Extensional abstractions They abstract instances rather than abstracting the language of terms. Note that when considering extensional abstractions, $\vee$ is the set theoretic union $\cup$, and that the order relation is the set theoretic inclusion.

Example 1. As an example consider $O=\{1,2,3,4\}$, and the abstraction $A$ obtained by closing under union the part $\{\{1,2\},\{1,3\}\}$ of $\mathcal{P}(O)$, so adding $\{1,2,3\}$ and $\emptyset$ to build $A$. The set of $\cup$-irreducible elements of $A$ is $A_{I}=\{\{1,2\},\{1,3\}\}$. For instance, $p_{A}(\{1,2,3\})=\{1,2\} \cup\{1,3\}=\{1,2,3\}$ as both elements of $A_{I}$ are included in $\{1,2,3\}$, and $p_{A}(\{2,3\})=\emptyset$ because neither $\{1,2\}$ nor $\{1,3\}$ is included in $\{2,3\}$.

We remark in the next section that abstractions are partially ordered.

\subsection{The lattice of abstractions}

There is a partial order on projections of a lattice $M[20]$ and therefore, on abstractions:

Definition 5 Let $M$ be a lattice and $p_{1}$ et $p_{2}$ two projections of $M$, we will state that $p_{2} \leq p_{1}$, i.e. $p_{2}$ is less concrete, and so more abstract than $p_{1}$, iff there is some projection $p$ defined on $p_{1}(M)$ such that for all $c$ in $M, p_{2}(c)=p \circ p_{1}(c)$.

This means that $A_{2}=p_{2}(M)$ is more abstract than $A_{1}=p_{1}(M)$ iff $A_{2}$ is an abstraction of $A_{1}$. This is also equivalent to saying that any $\vee$-irréductible element $i_{2}$ of $A_{2}$ may be written as a disjunction of $\vee$-irreducible elements of $A_{1}$, i.e. $i_{2}=i_{1}^{1} \vee \cdots \vee i_{1}^{n}$

\footnotetext{
${ }^{4}$ Irreducible elements of $A$ are elements that cannot be obtained as a result of applying $\vee_{M}$.
} 
Example 2 (Extensional abstractions). Let $M=\mathcal{P}(\{1,2,3,4\}), A_{I 1}=\{\{1,2\},\{1,3\}\}$ and $A_{2 I}=\{\{1,2\},\{1,2,3\}\}, A_{2}$ is more abstract than $A_{1}$ as $\{1,2\} \in A_{I 1}$ and $\{1,2,3\}=\{1,2\} \cup\{1,3\}$.

Proposition 5 (Lattice of abstractions) Let $M$ be a lattice, and $\mathcal{A}$ be the set of the abstractions of $M$. Let then $A_{1}=p_{1}(M)$ and $A_{2}=p_{2}(M)$ be elements of $\mathcal{A}$ :

- The least upper bound $A_{1} \vee_{\mathcal{A}} A_{2}$ is obtained by closing $A_{1} \cup A_{2}$ under $\vee$.

- The greatest lower bound $A_{1} \wedge_{\mathcal{A}} A_{2}$ is simply $A_{1} \cap A_{2}$.

This partial order, defined through projections, is known to order projected extensionintension lattices [20]. Therefore, abstract extension-intension lattices, where abstraction is performed on $\mathcal{L}$ or $\mathcal{P}(O)$, are also ordered following the lattice of abstractions.

\section{Extensional abstractions and extensional abstract lattices}

\subsection{Extensional abstract lattices}

An extensional abstraction is obtained by considering part of the powerset of $O$, and closing it by the union operator $\cup$. This means that we do not consider any more instances but rather subsets of instances, called abstract instances.

Definition 6 Let $A$ be an extensional abstraction and $p_{A}$ the associated projection of $\mathcal{P}(O)$, then let $t$ be a term of $\mathcal{L}$, ext $A_{A}(t)=p_{A} \circ \operatorname{ext}(t)$ is the abstract extension of $t$.

From Proposition 4 we also have

$$
\operatorname{ext}_{A}(t)=\bigcup_{u \in A_{I}, u \subseteq \operatorname{ext}(t)} u
$$

Definition 7 Let $A$ be an abstraction of $\mathcal{P}(O)$, the projected extension-intension lattice $p_{A}(G)$ is denoted as an extensional abstract lattice, and noted $G_{A}$.

Hereunder we define abstract instances and minimal abstractions of a given instance.

Definition 8 Let $A$ be an extensional abstraction.

- An element of $A_{I}$ is called an abstract instance.

- Let $A_{I}(o)$ be the subset of $A_{I}$ whose elements contain the instance o. We denote as minimal abstractions of an instance o the minimal elements of $A_{I}(o)$, i.e. $A_{m}(o)=$ $\left\{u \in A_{I} \mid o \in u\right.$ and if $o \in u^{\prime} \subset u$, then $\left.u^{\prime} \notin A\right\}$.

An interesting point is that $A_{I}$ is the set of minimal abstractions of the instances:

\section{Proposition 6}

$$
A_{I}=\bigcup_{\{o \in O\}} A_{m}(o)
$$


Example 3. In this example $O=\{1,2,3,4\}$ and $A_{I}=\{\{1,2\},\{2,3\},\{3,4\}\}$. Here $\mathcal{L}=\mathcal{P}(P)$ where $P=\{X, Y, Z\}$ is a set of properties. We give hereunder a table representing the context relating $\mathcal{P}(P)$ to $\mathcal{P}(O)$. We have added a column for each element of $A_{I}$. In Figure 1 we have represented the original concept lattice together with the extensional abstract lattice (here an abstract concept lattice) associated to $A_{I}$.

\begin{tabular}{|l|l|l|l|l|l|l|}
\hline Instances & $X$ & $Y$ & $Z$ & $\{1,2\}$ & $\{2,3\}$ & $\{3,4\}$ \\
\hline 1 & 1 & 0 & 1 & 1 & 0 & 0 \\
2 & 1 & 1 & 1 & 1 & 1 & 0 \\
3 & 0 & 1 & 0 & 0 & 1 & 1 \\
4 & 1 & 0 & 0 & 0 & 0 & 1 \\
\hline
\end{tabular}
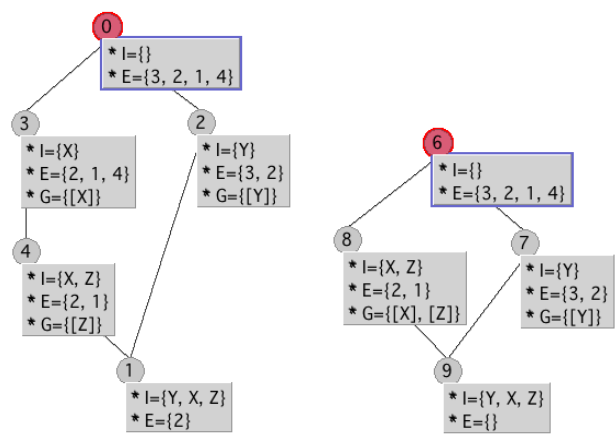

Fig. 1. The concept lattice (on the left) and the abstract concept lattice (on the right) corresponding to the context and abstraction given in Example 3. On each node $I$ is the intension, $E$ is the extension, and $G$ is the set of most general terms whose extension is $E$. The nodes 3 and 4 of the original concept lattice are merged into the node 8 of the abstract concept lattice. As a result, the abstract implication $X \rightarrow Z$ is now valid as $X$ and $Z$ have the same abstract extension.

We may note that such an extensional abstract lattice is isomorphic to an extensionintension lattice relating $\mathcal{L}$ to the powerset of $A_{I}$. For that purpose, we reformulate $A_{I}$ as a new instance set and we define $\left(e x t^{\prime}: \mathcal{L} \rightarrow A_{I}\right)$ as follows: let $u=\left\{o_{1}, \ldots o_{k}\right\}$ be an element of $A_{I}$, we have then that $u$, as a new instance, belongs to $\operatorname{ext}^{\prime}(t)$ whenever $u$, as a subset of $O$, is included in $\operatorname{ext}(t)$. As a consequence $\operatorname{ext}_{A}(t)=\bigcup_{u \in e x t^{\prime}(t)} u$ and therefore the images $\operatorname{ext}^{\prime}(\mathcal{L})$ and $\operatorname{ext}_{A}(\mathcal{L})$ are in one to one correspondence.

As stated before, an extension-intension lattice corresponds to a set of valid implications. We define hereunder the abstract implications associated to an extensional abstract lattice.

Definition 9 (Abstract implication) Let $t_{1}$ et $t_{2}$ be terms of $\mathcal{L}$. Whenever ext $t_{A}\left(t_{1}\right) \subseteq$ $\operatorname{ext}_{A}\left(t_{2}\right)$ we say that the abstract implication $t_{1} \rightarrow_{A} t_{2}$ is valid on $A$.

Then $G_{A}$ is represented by the whole set of abstract implications valid on $A$, or by any generating subset, as the min-max basis of abstract implications extending to the 
extensional abstract lattices the definition of the min-max basis of implications (see end of Section 2.2).

Now as $t_{1} \rightarrow t_{2}$ means that $\operatorname{ext}\left(t_{1}\right) \subseteq \operatorname{ext}\left(t_{2}\right)$, we have that whenever some abstract instance $u$ is included in $\operatorname{ext}\left(t_{1}\right)$, we also have that $u$ is included in $\operatorname{ext}\left(t_{2}\right)$. This also means, by definition, that $\operatorname{ext}_{A}\left(t_{1}\right)$ is included in $\operatorname{ext}_{A}\left(t_{2}\right)$. As a consequence validity of implications is preserved by extensional abstraction:

Proposition 7 Let $A$ be an abstraction of $\mathcal{P}(O)$, and $t_{1}$ and $t_{2}$ two terms of $\mathcal{L}$. If $t_{1} \rightarrow t_{2}$ is valid on $O$, then the abstract implication $t_{1} \rightarrow_{A} t_{2}$ is valid on $A$.

\subsection{Alpha lattices as extensional abstract lattices}

The partial order of abstractions (see Definition 5), means that $A^{\prime}$ is more abstract than $A$ whenever any element of $A^{\prime}$ (or $A_{I}^{\prime}$ ) may be written as the union of elements of $A$ (or $A_{I}$ ). An interesting case is alpha abstraction. Starting from a subset $\mathcal{C}$ whose union closure is an initial abstraction $A$, more concrete abstractions $A^{\alpha}$ are built. $A^{\alpha}$ is obtained by deriving from each initial category $C$ the set of frequent enough parts of $C$, and closing under union the resulting set $\mathcal{C}^{\alpha}$. We have then that $A^{\alpha}$ is more abstract than $A^{\alpha^{\prime}}$ iff $\alpha \geq \alpha^{\prime}$. In a previous work the corresponding alpha Galois lattices were defined through projections and experimented in order to extract alpha association rules [28]. As an example, consider the implication "Animals that fly are oviparous", it is not valid on $O$ because of the bat. When considering the categorization $\mathcal{C}=\{$ mammal, insect, bird $\}$, the corresponding abstract implication is valid but never applies: none of the initial categories contains only flying animals. However by considering frequent enough parts of each category, the corresponding $(\alpha=0.1)$ - implication is valid, as the bat is eliminated from the premise (very few mammals fly), but still applies to flying birds and flying insects, as they represent large enough parts of their categories.

\section{The extended abstract lattice $G_{\square}$}

We will formalize the nature of abstract implications by interpreting them as classical implications relating modalized terms. So we rewrite $t_{1} \rightarrow_{A} t_{2}$ as $\square_{A} t_{1} \rightarrow \square_{A} t_{2}$. We first define a modal logics of abstraction built on a propositional modal language $\mathcal{L}_{\text {mod }}$. Then we restrict $\mathcal{L}_{\text {mod }}$ to a language $\mathcal{L} \square$ using only conjunction and non nested modal connectors. Finally we present an extended abstract lattice $G_{\square}$ where intensions have both modalized and non modalized parts.

\subsection{Modal logics of abstraction}

Hereunder we discuss the modal logics of abstractions. Note that in this section we denote as worlds the elements of $O$.

A modal logic, in its simplest form, is a propositional logic to which is added at least one unary modal connector $\square$, referred to as a necessity operator. Classical modal logics are the modal logics in which formulas are given truth values through neighborhood semantics, also known as minimal models semantics [7]. This wide class of modal logics 
includes in particular normal modal logics relying on Kripke possible world semantics. We will define hereunder abstract modal logics as particular classical modal logics. We hereunder very informally summarize neighborhood semantics in order to relate such semantics to our extensional abstractions.

The modal language $\mathcal{L}_{\text {mod }}$ is obtained by adding the modal connector $\square$ to a propositional language $\mathcal{L}$ built on a set of atomic propositions. So for instance, $\phi=a \wedge \square(b \wedge$ $c$ ) belongs to $\mathcal{L}_{\text {mod }}$. In order to give a truth value to a modal formula we first consider a set of worlds $O$, together with a valuation function ext, relating each atomic proposition $p$ to the set of worlds in which $p$ is true. For any formula $\phi$ without modal connectors, the computation of $\operatorname{ext}(\phi)$ is then straightforward, for instance $\operatorname{ext}(a \wedge b)=\operatorname{ext}(a) \cap \operatorname{ext}(b)$ and represents the worlds $o$ in which $\phi$ is true, or in other words, the worlds that satisfies $\phi$. In order to extend ext to modal formulas we need a neighboring function $\mathcal{N}$ relating each world $o$ to a set of subsets of $O$ and we say that the world $o$ is in $\operatorname{ext}(\square \phi)$ whenever $\operatorname{ext}(\phi)$ belongs to $\mathcal{N}(o)$. It is known that neighboring functions $\mathcal{N}$ are in one to one correspondence with mappings $m: \mathcal{P}(O) \rightarrow \mathcal{P}(O)$ such that $m(e)=\{o \in O \mid e \in \mathcal{N}(o)\}$. To summarize we have now that

$$
\operatorname{ext}(\square \phi)=m \circ \operatorname{ext}(\phi)
$$

Now recall that we defined abstract extension as $p \circ \operatorname{ext}$ where $p$ is a projection. We will so naturally define abstract modal logics as classical modal logics in which the mapping $m$ is a projection. To characterize abstract modal logics we have just to translate the properties of projection as axioms and inference rules. Detailed results on modal logics of abstraction, including multimodal logics allowing to access to various levels of abstraction, are outside the scope of this paper [22]. Now, considering the abstract extension of a term as the extension of an abstract term leads to define extension-intension built on languages whose terms contain both non abstract and abstract parts. This is the subject of the remaining of this section.

\subsection{Mixing abstract and non abstract statements: the language $\mathcal{L} \square$}

We consider here a language $\mathcal{L} \square$ where classical properties and modalized properties appear simultaneously. Technically $\mathcal{L} \square$ is a subset of $\mathcal{L}_{\text {mod }}$ whose terms contain $\wedge$ and $\square$ as connectors, and in which the nesting of $\square$ is not allowed. For instance, $(a \wedge b) \wedge \square(b \wedge c) \wedge \square(c \wedge d) \in \mathcal{L} \square$, but $\square((a \wedge b) \wedge \square(b \wedge c)) \notin \mathcal{L} \square$.

We first give an inductive definition of $\mathcal{L} \square$.

Definition 10 ( Inductive definition of $\mathcal{L}_{P}$ and $\mathcal{L} \square$ ) The inductive definition of $\mathcal{L} \square$ according to $\mathcal{P}$ a non-empty set of atoms is the following (note that we define and use the language $\mathcal{L}_{P}$ corresponding to terms without any $\square$ connective):

- $\forall a \in \mathcal{P}, a \in \mathcal{L}_{P}$

- Constants $\top$ and $\perp \in \mathcal{L}_{P}$

- if F1 and $F 2 \in \mathcal{L}_{P}$ then $(F 1 \wedge F 2) \in \mathcal{L}_{P}$

- if $F \in \mathcal{L}_{P}$ then $F \in \mathcal{L} \square$

- if $F \in \mathcal{L}_{P}$ then $\square F \in \mathcal{L} \square$

- if $F 1 \wedge F 2 \in \mathcal{L} \square$ then $(F 1 \wedge F 2) \in \mathcal{L} \square$ 


\section{An intensional semantics for $\mathcal{L} \square$}

We define an intensional semantics for $\mathcal{L} \square$ based on an algebraic approach similar to [27], [10] but for description logics. The definition is made in two steps. In the first step, an equational system which highlights the main properties of the $\mathcal{L} \square$ connectives is given. During the second step, an homomorphism based on the equational system is defined. This homomorphism is used to map terms of $\mathcal{L} \square$ to their structural normal form (snf) in the intensional semantics.

\section{Definition 11 ( The equational system $E Q_{\square}$ ) $\forall F 1, F 2, F 3 \in \mathcal{L} \square, \forall F, F^{\prime} \in \mathcal{L}_{P}$ :}

1. $(F 1 \wedge F 2) \wedge F 3=F 1 \wedge(F 2 \wedge F 3)$

2. $F 1 \wedge F 2=F 2 \wedge F 1$

3. $F 1 \wedge F 1=F 1$

4. $\top \wedge F 1=F 1$

5. $\perp \wedge F 1=\perp$

6. $\square F=\square F \wedge F$

7. $\square\left(F \wedge F^{\prime}\right)=\square\left(F \wedge F^{\prime}\right) \wedge \square F \wedge \square F^{\prime}$

The equational system $E Q_{\square}$ fixes the main properties of the connectives and can be used to define an equivalence relation between terms of $\mathcal{L} \square$. Equality modulo axioms of $E Q_{\square}$ is denoted by $\equiv_{E Q_{\square}}$. We use it to formalize the subsumption relation in $\mathcal{L} \square$.

Definition 12 (Subsumption in $\mathcal{L} \square$ ) Let $F 1$, and $F 2$ be two terms of $\mathcal{L} \square, F 1 \preceq F 2$ (i.e. F1 subsumes or is less specific than F2) iff $F 1 \wedge F 2 \equiv_{E Q_{\square}} F 2$.

$E Q_{\square}$ induces a class of algebras. From this class, a structural algebra can be proposed, which provides $\mathcal{L} \square$ with an intensional semantics called $\mathcal{C} \mathcal{L} \square$. The elements of $\mathcal{C} \mathcal{L} \square$ are structures whose definition is given below. These elements are structural normal forms of terms of $\mathcal{L} \square$ allowing us to obtain an unique class representative for each equivalence classes of terms. $\mathcal{C} \mathcal{L} \square$ can be viewed as a normalized subset of $\mathcal{L} \square$ where the $\operatorname{lgg}$ is unique.

\section{The intensional semantics $\mathcal{C} \mathcal{L} \square$}

An element of $\mathcal{C} \mathcal{L} \square$ corresponding to a term $T$ of $\mathcal{L} \square$ denoted $\operatorname{snf}(T)$ is a pair defined as follows: $\langle$ Eclassical, $E \square>$. Eclassical is a set of atoms belonging to $\mathcal{P}, E \square$ is a set of subsets of $\mathcal{P}$. Intuitively, Eclassical contains every explicit and implicit classical properties of $T$ (i.e. properties not at reach to a $\square$ connective). The data structure used can then be a simple set of atoms. $E \square$ contains $\square$ properties of $T$. Since for instance $\square(a \wedge b)$ and $\square(b \wedge d)$ cannot be compared, we must keep both of them. The data structure used is a set of sets.

This definition presents the data structure of elements of $\mathcal{C} \mathcal{L} \square$ but not how to associate to a term of $\mathcal{L} \square$ its corresponding element in $\mathcal{C} \mathcal{L} \square$. To make this computation and then to define $\mathcal{C} \mathcal{L} \square$, an homomorphism from the set of terms of $\mathcal{L} \square$ and the set of elements of $\mathcal{C L} \square$ need to be defined.

The homomorphism from $\mathcal{L} \square$ into $\mathcal{C} \mathcal{L} \square$ is sketched below. It allows us to associate to each term $T$ of $\mathcal{L} \square$ its structural normal form denoted $\operatorname{snf}(T)$. This homoporphism takes into account axioms of $E Q_{\square}$ and the normalization strategy choosen. Indeed, to 
obtain a normal form many normalization strategies may be applied (e.g. deletion of redundant information). We chose to add implicit information in the classical part, this strategy is a kind of partial saturation which is a trick largely used to make easier subsumption and lgg computation. On the other hand, for complexity reasons we only keep maximal subsets in the $\square$ part. The $\square$ part is a Sperner family i.e., an antichain in the inclusion lattice over the power set of $\mathcal{L}_{P}$ (for more details see [2]).

\section{Homomorphism snf from $\mathcal{L} \square$ into $\mathcal{C} \mathcal{L} \square$ :}

$$
\begin{array}{c|c|}
\text { Term of } \mathcal{L} \square & \text { Element in } \mathcal{C} \mathcal{L} \square \\
\top & t=<\emptyset,\{\emptyset\}> \\
\perp & b o=<\mathcal{P},\{\mathcal{P}\}> \\
a \in \mathcal{P} & <\{\mathrm{a}\},\{\emptyset\}> \\
F 1 \wedge F 2 & \operatorname{snf}(F 1) \vee_{s} \operatorname{snf}(F 2) \\
\square F & <E_{F},\left\{E_{F}\right\}>
\end{array}
$$

where $\operatorname{snf}(F)=<E_{F}, \emptyset>$ (the $\square$ part is empty since there is no $\square$ nesting).

$\vee_{s}$ is the join operator in $\mathcal{C} \mathcal{L} \square$. It uses the classical union set operator and $\vee_{a}$ which represents the union operator in antichains.

Let $K 1$ and $K 2$ be two antichains:

$$
K 1 \vee_{a} K 2=\{x \in K 1 \cup K 2 \mid \nexists y \in K 1 \cup K 2 \text { s.t. } x \subset y\}
$$

Let $\operatorname{snf}(F i)$ be $<E_{F i}, K i>$ for $i=1,2$ :

$$
\operatorname{snf}(F 1) \vee_{s} \operatorname{snf}(F 2)=<E_{F 1} \cup E_{F 2}, K 1 \vee_{a} K 2>
$$

Example 4. $\operatorname{snf}((a \wedge b) \wedge \square(b \wedge c) \wedge \square b \wedge \square(c \wedge d))=<\{a, b, c, d\},\{\{b, c\},\{c, d\}\}>$ $c$ and $d$ are added in the classical part according to axiom 6 and $7,\{b\}$ is removed from the $\square$ part since $\{b\} \subseteq\{b, c\}$.

Definition 13 (Structural Subsumption in $\mathcal{C} \mathcal{L} \square$ ) Let $S 1$ and $S 2$ be two elements of $\mathcal{C} \mathcal{L} \square$,

$$
S 1 \preceq_{s} S 2 \text { (i.e. } S 1 \text { subsumes } S 2 \text { ) iff } S 1 \vee_{s} S 2=S 2
$$

Proposition 8 Subsumption in $\mathcal{L} \square$ is equivalent to structural Subsumption in $\mathcal{C} \mathcal{L} \square$ : Let $F 1$, and $F 2$ be two terms of $\mathcal{L} \square$,

$$
F 1 \preceq F 2 \text { iff } \operatorname{snf}(F 1) \preceq s \operatorname{snf}(F 2)
$$

\subsection{The Galois lattice $\mathbf{G}_{\square}$}

We consider the two following posets : $(\mathcal{P}(O), \subseteq)$ and $\left(\mathcal{C} \mathcal{L} \square, \preceq_{s}\right)$. In order to obtain a Galois connection between the two posets, we need to define the least general generalisation in $\mathcal{C} \mathcal{L} \square$. The $\operatorname{lgg}$ uses the following definition of $\wedge_{a}$ the intersection operator in 
antichain: any antichain $A$ corresponds to a lower set $L_{A}=\{x \in A \mid \exists y \in A$ s.t. $x \subseteq$ $y$ \}. Let $A$ and $B$ be two antichains and $L_{A}$ and $L_{B}$ their lower sets:

$$
A \wedge_{a} B=\left\{x \in L_{A} \cap L_{B} \mid \nexists y \in L_{A} \cap L_{B} \text { s.t. } x \subset y\right\}
$$

Definition 14 (Least General Generalisation in $\mathcal{C} \mathcal{L} \square$ ) Let $S 1=\langle E 1, K 1>$ and $S 2$ $=<E 2, K 2>$ be two elements in $\mathcal{C} \mathcal{L} \square$ :

$$
\operatorname{lgg}(S 1, S 2)=<E 1 \cap E 2, K 1 \wedge_{a} K 2>
$$

Let EnsS be a finite set of elements of $\mathcal{C} \mathcal{L} \square=\{s 1, s 2, \ldots, s n\}$ :

$$
\operatorname{lgg}(\operatorname{Ens} S)=\operatorname{lgg}(s 1, \operatorname{lgg}(s 2, \ldots \lg (s n-1, s n)))
$$

Each instance $o$ is described by a term $T$ of $\mathcal{L} \square$. In order to obtain a unique most specific description in $\mathcal{C} \mathcal{L} \square$ satisfied by $o$, we simply use $\operatorname{snf}(T)$ denoted $d(o)$ in the following.

Proposition 9 Let $\left(\mathcal{C} L \square, \preceq_{s}\right)$ and $(\mathcal{P}(O), \subseteq)$ be two posets. The pair (ext,int) defined by:

$$
\begin{gathered}
\forall S \in \mathcal{C} \mathcal{L} \square, \operatorname{ext}(S)=\{o \in O \mid S \preceq s d(o)\} \\
\forall E \in \mathcal{P}(O), \operatorname{int}(E)=\operatorname{lgg}(d(E)) \text { with } d(E)=\{d(o) \mid \forall o \in E\}
\end{gathered}
$$

defines a Galois connection between $\left(\mathcal{C} \mathcal{L} \square, \preceq_{s}\right)$ and $(\mathcal{P}(O), \subseteq)$

Proof : as stated in proposition 4 p11 in [15], (ext,int) is a Galois connection iff:

$$
S \preceq_{s} \operatorname{int}(E) \Leftrightarrow E \subseteq \operatorname{ext}(S)
$$

1) $S \preceq_{s} \operatorname{int}(E) \Rightarrow S \preceq_{s} \operatorname{lgg}(d(E))$

$\forall o \in E, \operatorname{lgg}(d(E)) \preceq_{s} d(o)$ since $\operatorname{lgg}(d(E))=\operatorname{lgg}(d(o), \operatorname{lgg}(d(E-\{o\}))$

$\Rightarrow S \preceq_{s} d(o)$ since $S \preceq_{s} \operatorname{lgg}(d(E)) \Rightarrow o \in \operatorname{ext}(S)$ by definition of ext $\Rightarrow E \subseteq \operatorname{ext}(S)$

2) $E \subseteq \operatorname{ext}(S) \Rightarrow(\forall o \in E \Rightarrow o \in \operatorname{ext}(S)) \Rightarrow \forall o \in E, S \preceq_{s} d(o) \Rightarrow S \preceq_{s} \operatorname{int}(E)$

Proposition 10 The extension-intension lattice $G_{\square}$ defined by the Galois connection of proposition 9 is denoted as an extended abstract lattice.

When considering a given extensional abstraction $A$, we have now a "pure" extensional abstract lattice $G_{A}$, as defined in section 4 together with the new intension/extension lattice $G_{\square}$ defined on the connection between $\mathcal{C} \mathcal{L} \square$ and $\mathcal{P}(O)$ and relying, for its modalized part, on $\operatorname{ext}(\square t)=p_{A} \circ \operatorname{ext}(t)$. We are now interested in the exact relations between these lattices and the original concept lattice $G$ relating the language $\mathcal{P}(P)$ to $\mathcal{P}(O)$. We first remark that $\mathcal{P}(P)$ is isomorphic to an abstraction of $\mathcal{C} \mathcal{L} \square$ as the classical part of $\mathcal{C L} \square$ is closed under the lgg operator. Therefore $G$ is obtained as an intensional abstraction of $G_{\square}$. A second remark is that the extensional abstract lattice $p_{A}\left(G_{\square}\right)$ is isomorphic to $G_{A}$ : consider some intension $t$ in $G$, its abstract extension $e=\operatorname{ext}_{A}(t)$ rewrites as $\operatorname{ext}(\square t)$, and the representation of $\square t$ is the most specific element in $\mathcal{C} \mathcal{L} \square$ whose extension contains $e$. As a consequence, $G_{A}$ and $G$ are both less abstract than $G_{\square}$. We draw Figure 2 these three lattices in a very simple example with two atomic properties $X$ and $Y, 4$ instances and the following abstraction $A_{I}=\{\{1,2\},\{2,3\},\{3,4\}\}$. Note that in the table hereunder, the columns $\square t$ represent the abstract extensions $\operatorname{ext}_{A}(t)$. For instance the column $\square X Y$ represents the 
empty set since $\operatorname{ext}(X Y)=\{2\}$ and that no abstract instance of $A_{I}$ is included in $\operatorname{ext}(X Y)$.

\begin{tabular}{|l|l|l|l|l|l|l|l|l|}
\hline Instance & $\mathrm{X}$ & $\mathrm{Y}$ & $\square \mathrm{X}$ & $\square \mathrm{Y}$ & $\square \mathrm{XY}$ & $\{1,2\}$ & $\{2,3\}$ & $\{3,4\}$ \\
\hline 1 & 1 & 0 & 1 & 0 & 0 & 1 & 0 & 0 \\
2 & 1 & 1 & 1 & 1 & 0 & 1 & 1 & 0 \\
3 & 0 & 1 & 0 & 1 & 0 & 0 & 1 & 1 \\
4 & 1 & 0 & 0 & 0 & 0 & 0 & 0 & 1 \\
\hline
\end{tabular}
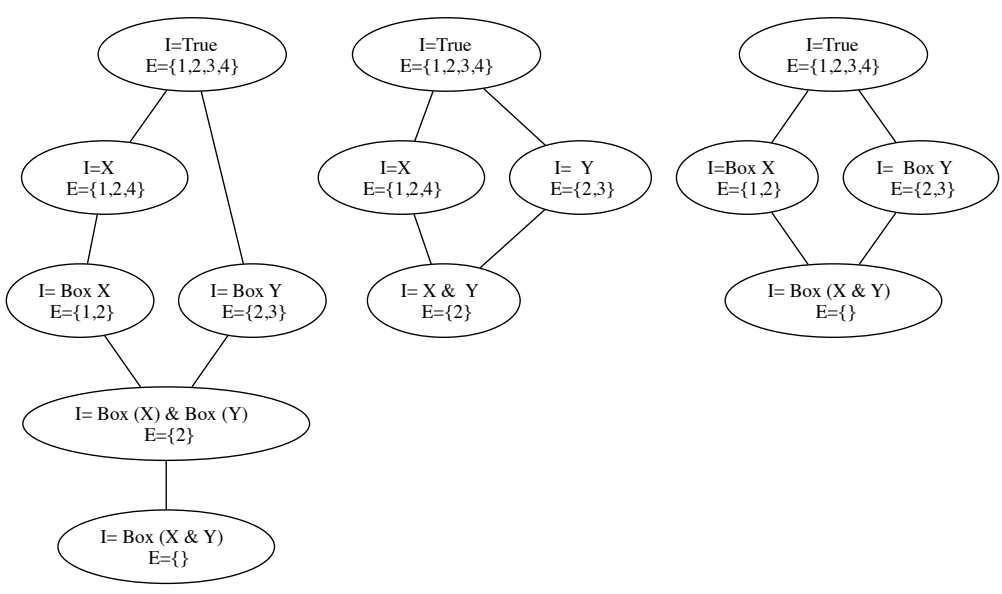

Fig. 2. The three intension/extension lattices corresponding to the example of section 5.2. The node label is a minimal representation of the intension $I$ (here Box and \& stand for $\square$ and $\wedge$ ) together with its extension $E$ in $\{1,2,3,4\}$. The leftmost lattice is $G_{\square}$, the centermost lattice is the original concept lattice $G$, and the rightmost lattice is the projected lattice $p_{A}\left(G_{\square}\right)$ isomorphic to the abstract lattice $G_{A}$

\section{Related work and conclusion}

In this paper we have proposed abstractions as reductions that preserve the extensionintension lattice structure. They are simply defined as parts of either the intensional language or the extensional space that are closed under the join operator. A second contribution is the investigation of extensional abstractions. We have shown that defining an extensional abstraction $A \subseteq \mathcal{P}(O)$ consists in a priori defining as units particular subsets of instances, denoted as abstract instances, so applying a change in extensional granularity. A noticeable effect of so preserving the Galois lattice structure is that validity of implications is preserved through extensional abstractions. Finally, we interpret abstract implications as classical implications between two modalized terms. This leads to define abstract modal logics and to define an extended abstract concept lattice relating $\mathcal{P}(O)$ to a language whose elements have abstract and non abstract parts. We can 
then search for concepts as "Oviparous and abstractly apt-to-fly" where "o satifies $a b$ stractly apt-to fly" means that all the instances of some minimal abstraction of $o$ share this property. Regarding the implementation of abstract concept lattice, we can benefit from implementations of alpha latices, and in particular their incremental construction [23] adapted from [26], and a software, based on Galicia [24], is available ${ }^{5}$ However, extended abstract concept lattice construction still has to be investigated, both from an algorithmic and practical point of view. Note that as extended abstract lattices belongs to the class of logical concept lattices [13] yet there exists a way to implement them.

Acknowledgments We are grateful to Auguste Forge for useful comments and careful reading of this paper.

\section{References}

1. Shai Ben-David and Rachel Ben-Eliyahu-Zohary. A modal logic for subjective default reasoning. Artif. Intell., 116(1-2):217-236, 2000.

2. Béla Bollobás. Combinatorics: set systems, hypergraphs, families of vectors, and combinatorial probability. Cambridge University Press, New York, NY, USA, 1986.

3. P. Brézellec and Henry Soldano. Tabata: a learning algorithm performing a bidirectional search in a reduced search space using a tabu stratégie. In Eur. Conf. in Art. Int., ECAI-98, pages 420-424, Brighton, England, 1998. Wiley and Sons.

4. Nathalie Caspard and Bernard Monjardet. The lattices of closure systems, closure operators, and implicational systems on a finite set: a survey. Discrete Appl. Math., 127(2):241-269, 2003.

5. Gianpiero Cattaneo and Davide Ciucci. Lattices with interior and closure operators and abstract approximation spaces. T. Rough Sets, 10:67-116, 2009.

6. Laurent Chaudron and Nicolas Maille. Generalized formal concept analysis. In Bernhard Ganter and Guy W. Mineau, editors, ICCS, volume 1867 of Lecture Notes in Computer Science, pages 357-370. Springer, 2000.

7. Brian F. Chellas. Modal Logic: an introduction. Cambridge University Press, 1980.

8. B. Davvaz and M. Mahdavipour. Rough approximations in a general approximation space and their fundamental properties. International Journal of General Systems, 37(3):373 386, 2008.

9. Edwin Diday and Richard Emilion. Maximal and stochastic galois lattices. Discrete Appl. Math., 127(2):271-284, 2003.

10. Robert Dionne, Eric Mays, and Frank J. Oles. The equivalence of model-theoretic and structural subsumption in description logics. In Int. Joint Conf. on Art. Int. (IJCAI), pages 710717, 1993.

11. M. Erné, J. Koslowski, A. Melton, and G. E. Strecker. A Primer on Galois Connections. Annals of the New York Academy of Sciences, 704(Papers on General Topology and Applications):103-125, 1993.

12. S. Ferré. Negation, opposition, and possibility in logical concept analysis. In Rokia Missaoui and $\mathrm{J}$ rg Schmid, editors, Int. Conf. Formal Concept Analysis, LNCS 3874, pages 130-145. Springer, 2006.

$\overline{5}$ http://www-lipn.univ-paris13.fr/ champesme/alphabetagalicia. 
13. S. Ferré and O. Ridoux. An introduction to logical information systems. Information Processing and Management, 40(3):383-419, 2004.

14. J-G. Ganascia. TDIS: an algebraic formalization. In Int. Joint Conf. on Art. Int. (IJCAI), volume 2, pages 1008-1013, 1993.

15. B. Ganter and R. Wille. Formal Concept Analysis: Mathematical Foundations. Springer Verlag, 1999.

16. Bernhard Ganter and Sergei O. Kuznetsov. Pattern structures and their projections. ICCS-01, LNCS, 2120:129-142, 2001.

17. J.L. Guigues and V. Duquenne. Famille non redondante d'implications informatives résultant d'un tableau de données binaires. Mathématiques et Sciences humaines, 95:5-18, 1986.

18. Michel Liquière and Jean Sallantin. Structural machine learning with galois lattice and graphs. In ICML '98: Proceedings of the Fifteenth International Conference on Machine Learning, pages 305-313, San Francisco, CA, USA, 1998. Morgan Kaufmann Publishers Inc.

19. Nicolas Pasquier, Rafik Taouil, Yves Bastide, Gerd Stumme, and Lotfi Lakhal. Generating a condensed representation for association rules. Journal Intelligent Information Systems (JIIS), 24(1):29-60, 2005.

20. Nathalie Pernelle, Marie-Christine Rousset, Henry Soldano, and Véronique Ventos. Zoom: a nested Galois lattices-based system for conceptual clustering. J. of Experimental and Theoretical Artificial Intelligence, 2/3(14):157-187, 2002.

21. Lorenza Saitta and Jean-Daniel Zucker. A model of abstraction in visual perception. Applied Artificial Intelligence, 15(8):761-776, 2001.

22. Henry Soldano. A modal view on abstract learning and reasoning. Technical report, L.I.P.N, UMR-CNRS 7030, Université Paris-Nord, 2010.

23. Henry Soldano, Véronique Ventos, Marc Champesme, and David Forge. Incremental construction of alpha lattices and association rules. In Knowledge-Based and Intelligent Information and Engineering Systems, volume 6277 of Lecture Notes in Computer Science, pages 351-360. Springer Berlin / Heidelberg, 2010.

24. Petko Valtchev, David Grosser, Cyril Roume, and Mohamed Rouane Hacene. Galicia: An open platform for lattices. In In Using Conceptual Structures: Contributions to the 11th Intl. Conference on Conceptual Structures (ICCS'03, pages 241-254. Shaker Verlag, 2003.

25. Petko Valtchev, Rokia Missaoui, and Robert Godin. Formal concept analysis for knowledge discovery and data mining: The new challenges. In Peter W. Eklund, editor, ICFCA, volume 2961 of Lecture Notes in Computer Science, pages 352-371. Springer, 2004.

26. Petko Valtchev, Rokia Missaoui, Robert Godin, and Mohamed Meridji. Generating frequent itemsets incrementally: two novel approaches based on Galois lattice theory. J. of Experimental and Theoretical Artificial Intelligence, 2/3(14):115-142, 2002.

27. Véronique Ventos. A deductive study of the c-classicde description logic. In Description Logics, volume WS-96-05 of AAAI Technical Report, pages 192-196. AAAI Press, 1996.

28. Véronique Ventos and Henry Soldano. Alpha galois lattices: An overview. Proceedings of ICFCA'05, Lecture Notes on Computer Science, 3403:298-313, 2005.

29. Y. Y. Yao and T. Y. Lin. Generalization of rough sets using modal logics. Intelligent Automation and Soft Computing, an International Journal, 2:103-120, 1996. 